\title{
Practical Aspects in the Application of the New Legislation: Alternative Methods and 3R Principle, Role of Ethical Committees and Animal Suffering
}

\author{
Francesca Caloni ${ }^{1}$, Patrizia Costa ${ }^{2}$, Gianni Dal Negro ${ }^{3}$, Isabella De Angelis ${ }^{4}$, \\ Giovanna Lazzari ${ }^{5}$, Giacomo Metteucci ${ }^{6}$, and Augusto Vitale ${ }^{7}$ \\ ${ }^{1}$ Università degli Studi di Milano, Department of Health, Animal Science and Food Safety, Milan, Italy; ${ }^{2}$ Università degli \\ Studi di Milano, Laboratory Animal Science and Medicine Specialization School, Milan, Italy; ${ }^{3}$ GlaxoSmithKline \\ R\&D Limited, UK; ${ }^{4}$ Istituto Superiore di Sanità, Department of Environment and Primary Prevention, Rome, Italy; \\ ${ }^{5}$ Avantea, Laboratory of Reproductive Technologies, Cremona, Italy; ${ }^{6}$ Università degli studi di Milano, LAS and Animal \\ Welfare Consultant, Milan, Italy; ${ }^{7}$ Istituto Superiore di Sanità, Department of Cell Biology and Neuroscience, Section \\ of Behavioural Neuroscience, Rome, Italy
}

The 2012 edition of the open conference on animal testing, organized by the Specialization School in Laboratory Animal Science and Medicine of the Milano University and the Istituto Zooprofilattico Sperimentale della Lombardia e dell'Emilia Romagna Brescia, was held in Gargnano on May 14-16, 2012. This edition focused on "Practical aspects in the application of the new legislation: alternative methods and $3 R$ principle, role of the committees and animal suffering," and it hosted a session completely dedicated to alternative approaches.

Sixteen speakers contributed to a two-day intense and interactive debate on the important aspects of the new directive on animal testing and research, within the context of the 3Rs principle.

The conference opened with an introduction to the Reference Center for Alternative Methods, Care and Welfare of Animals' activities, presented by Dr S. Cinotti, Dr M. Ferrari, and Dr G Lombardi.

The first session, dedicated to the role and responsibilities of the committees in the ethical review process with regard to the new Directive 2010/63/EU, featured speakers such as Dr G. Matteucci, Prof. A. Peli, (University of Bologna), and Dr L. Luperi (RTC). The second session, entitled "Refinement: established guidelines and experience in pain relief," was organized and moderated by Dr P. Costa in an effort to propose, disseminate, and consolidate such guidelines as
ARRIVE (Kilkenny et al., 2010) and Guidelines for the welfare and use of animals in cancer research (Workman et al., 2010), which have been established and shared by the scientific community. Speakers included Dr P. du Sert (NC3Rs), Dr O. Barbieri (University of Genoa), and Dr M. Campagnol (University of Milan).

As part of the third session, "Alternative $3 \mathrm{R}$ methods in practice," Dr P. Prieto (EURL-ECVAM) clearly illustrated the path that EURL-ECVAM (European Union Reference Laboratory for Alternatives to Animal Testing http://ihcp.jrc. ec.europa.eu/our_activities/alt-animal-testing) intends to follow in order to comply with the requirements specified by Directive 2010/63/EU. The mission of the reference laboratory of the EU is clearly defined by Annex VII "Duties and Tasks of the Union Reference Laboratory for the Validation of Alternative Methods." Of these duties, the following were underscored: i) promotion of the development and use of alternative methods, in both basic and applied research and in the regulatory field, ii) coordination of the process of validation of alternative methods at the European level. From this point of view, EURL-ECVAM is set to play a crucial role in the exchange of information on the development of alternatives and the promotion of dialogue between policy makers, the scientific community, and society. In order to achieve these objectives, EURL-ECVAM has certain operational tools at its dis- 
posal for consultation and experimentation, and for promotion and development, such as: ESAC (Scientific Committee of ECVAM, which states its opinion on the validity and relevance of an alternative method), PARERE (Preliminary Assessment of Regulatory Acceptance Network, a European network that provides input on the regulatory relevance of alternatives and works alongside ESAC), and ESTAF (ECVAM Stakeholder Forum, which maintains an open dialogue between the different components of the organization and gives voice to economic, social, and ethical interests). On the operating side, there is NETVAL (Network of Laboratories for the Validation of Alternative Methods), which comprises the laboratories, selected by the National Reference Centers, able to perform or coordinate validation studies based on their experience and expertise. Currently 37 laboratories from 12 European countries have become part of this network.

The next speaker, Dr K. Chapman (NC3Rs), explained the purpose, activities, and experience of the NC3Rs, the National Center for Alternatives to Animal Testing in the UK, reiterating that the creation and maintenance of a National Center always adds value to one's country. The NC3Rs, founded in 2004, works in collaboration with academia, industry, regulators, and animal welfare organizations, actively supporting the implementation and development of alternative methods. Specifically, the Center strives to motivate researchers to use the $3 \mathrm{Rs}$, to promote opportunities for funding research projects, and to the train young researchers. Other important activities of the NC3Rs include training and, above all, dissemination of data and information on the 3Rs. The creation of specific discussion forums and the organization of courses and symposiums are very important in this regard. One of the main initiatives to which the Center is committed, CRACK IT, aims through appropriate funding, to encourage the practical implementation of new ideas, technologies, and methodologies in the 3 Rs field by bringing together scientists from different disciplines.

Dr I. Ragan (European Partnership on Alternative Approaches to Animal Testing) then outlined the activities of EPAA regarding the quality control of vaccines. In the vaccine production process, about $80 \%$ of the animals (rodents) are used in the phase of quality control of all batches produced, immediately before market release (safety and potency tests). Although considerable progress has been made in recent years in the development and validation of alternative methods for this purpose, the quality control of vaccines is still a grey area in which the use of in vitro methods should become more frequent and widespread. EPAA is thus carrying out a project based on the "consistency" approach in order to implement and expand the use of these methods in this area. Briefly, the "consistency" approach provides for more careful checking of all the stages of vaccine production, including the selection of raw materials and the definition of a specific biological profile of the vaccine (fingerprint) that can serve as a check for all batches. The compliance of the different batches is then tested with in vitro methods. In addition to being a better approach from an ethical point of view, this method is also advanta- geous from the scientific (better characterization of the clinical batch) and economic (much faster quality control, a few days instead of two months) perspectives.

Finally, Dr M. Tollis (Istituto Superiore di Sanità) presented a series of considerations on the technical/regulatory requirements related to the application of alternative methods in the quality control of veterinary vaccines. This is an area in which a complex body of legislation (Directive 2001/82/EU and successive amendments [final 2009/9/EC] and monographs of the European Pharmacopoeia) has led to many opportunities in the use of alternative methods, especially through the optimization of assay conditions and better definition of the objectives to be pursued. It is not always necessary to demonstrate the effects of a vaccine by subjecting animals to experimental infections, the severity of which do not necessarily correspond to the actual severity of morbid symptoms observed in practice. Additionally, it is not always necessary to determine the maximum effectiveness of every batch produced, when the minimum conditions for the suitability of the vaccine have already been identified and established. Even for the purposes of checking the relative potency of a vaccine (batch potency test, BPT) the combination of in vivo and in vitro methods appears to allow for a significant reduction in the number of animals used. The validity of this approach has yet to be verified through a suitable validation process, however.

The presentation by Prof. F. Caloni (University of Milan) showed the use of the 3Rs (Replace, Reduce, Refine) in toxicology, research, and teaching (Daneshian et al., 2011). In vitro toxicology evaluates the effects of xenobiotics at the cellular and molecular level; in vitro methods that show remarkable reliability are then subjected to the process of validation before being used in the regulatory environment. There are validated in vitro assays that involve the use of reconstructed tissues or specific cell lines, but implementation is still necessary in areas such as toxicokinetics, repeated dose toxicity, skin sensitization, carcinogenicity, and reproductive toxicity. The concepts of the Integrated Testing Strategy (ITS) and evaluation of the Pathways of Toxicity (PoT) are important in this regard (Basketter et al., 2012). A multidisciplinary approach and ad hoc teaching (Caloni et al., 2011) are essential for increased application of the 3Rs in toxicology.

Dr G. Lazzari (Avantea, Cremona) explained the current state of development of alternative tests in the reproductive area. She first described the complexity of the reproductive cycle as an integrated physiological process that includes the development of the male and female gametes with supporting somatic cells, and the long process of development of the embryo that leads to pregnancy and the birth of the offspring. Dr Lazzari then described alternative tests that currently are published in the scientific literature, with particular reference to those already validated or in the process of being validated by ECVAM. She then elaborated on the different approaches to the development of alternative tests for reproductive toxicity, with particular reference to the results obtained in the framework of collaborative research projects funded by the $6^{\text {th }}$ and $7^{\text {th }}$ Framework Programs of the European Commis- 
sion. Finally, the speaker expounded on alternative tests developed by the Avantea laboratory using both bovine oocytes and pre-implantation embryos obtained through in vitro techniques, as well as embryonic stem cells. The oocyte is the cell in which transcripts and energy substrates are deposited and which lead to the initiation of embryonic development (Lazzari et al., 2008) with the formation of male and female pronuclei after fertilization, followed by the first embryonic divisions and the activation of the embryonic genome. For this reason, in vitro tests able to reveal significant negative effects on oocytes, early embryos, and embryonic stem cells are important components in the development of alternative tests for toxicity in the field of reproduction.

A practical example of the process of validation of an in vitro hematotoxicity assay was presented by Prof. A. Pessina (University of Milan). The in vitro CFU-GM clonogenic assay provides many benefits, such as improved definition of toxicological safety limits, contributing to the development of new therapeutic and 2R (Refine, Reduce) agents. Moreover, through a multidisciplinary approach and an innovative LoCMed project (Lab on Chip for testing Myelotoxic effect of drugs and chemicals, in collaboration with Politecnico di Milano (Bioengineering Department), the evaluation of clonogenicity can be performed using software for computerized data processing and analysis.

Dr V. Vasina (University of Bologna) addressed the application of in vitro methods in pharmacology, intended to complement and integrate with experimental in vivo models. Different in vitro (ex vivo) tools are used today, both for the study of inflammatory bowel diseases (IBD) and the preliminary screening of new drugs. This is an integrated strategy of in vivo-in vitro models, which allows for a reduction in the number of animals and a refinement of methodologies, in compliance with the principles of the 3 Rs.

Dr G. Dal Negro (GlaxoSmithKline, UK) described the application of alternative methods in pharmaceutical research and in other industrial sectors and pointed out that the current levels of scientific knowledge and technological development are not yet able to simulate the complexity of the living being or complex parts of it. To date, in spite of the scientific community and international authorities who strongly support the principle of reduction and, where possible, of replacement of animals with non-animal models, replacement is not yet pos- sible in many fields of application. The 3Rs-oriented initiatives should be more harmonized, and more emphasis should be given to efforts currently underway to refine and reduce the use of animals. An adequate information campaign to inform the community about the difficulties encountered and the progress made is now only in its infancy. In this regard, the activity of all organizations active in the field, both nationally and internationally (EPAA, EFPIA, FELASA), is extremely important.

\section{References}

Basketter, D. A., Clewell, H., Kimber, I., et al. (2012). A roadmap for the development of alternative (non-animal) methods for systemic toxicity testing. ALTEX 29, 5-91.

Caloni, F., Alberizzi, V., Costa, A., et al. (2011). Master students' feedback on 3Rs education approach. ALTEX 28, Spec Issue, 210

Daneshian, M., Akbarsha, M. A., Blaauboer, B., et al. (2011). A framework program for the teaching of alternative methods (replacement, reduction, refinement) to animal experimentation. ALTEX 28, 341-352.

Kilkenny, C, Browne, W. J., Cuthill, I. C., et al. (2010). Improving Bioscience Research Reporting: The ARRIVE Guidelines for Reporting Animal Research. PLoS Biol 8, e1000412.

Lazzari, G., Tessaro, I., Crotti, G., et al. (2008). Development of an in vitro test battery for assessing chemical effects on bovine germ cells under the ReProTect umbrella. Toxicol Appl Pharmacol 233, 360-370.

Workman, P., Aboagye, E. O., Balkwill, F., et al. (2010). Guidelines for the welfare and use of animals in cancer research. $\mathrm{Br}$ J Cancer 102, 1555-1577.

\section{Correspondence to}

\section{Francesca Caloni, DVM PhD}

Dipartimento VSA - Facoltà di Medicina Veterinaria

Università degli Studi di Milano

Via Celoria 10

20133 Milano

Italy

Phone: +39-02-503-17884

Email: francesca.caloni@unimi.it 